\title{
The prognostic significance of $p 16$ expression pattern in diffuse gliomas
}

\author{
Jin Woo Park ${ }^{1}$, Jeongwan Kang ${ }^{1}$, Ka Young Lim ${ }^{1}$, Hyunhee Kim ${ }^{1}$, Seong-Ik Kim \\ Jae Kyung Won ${ }^{1}$, Chul-Kee Park ${ }^{2}$, Sung-Hye Park ${ }^{1,3}$ \\ Departments of ${ }^{1}$ Pathology and ${ }^{2}$ Neurosurgery, Seoul National University Hospital, Seoul; \\ ${ }^{3}$ Neuroscience Research Institute, Seoul National University College of Medicine, Seoul, Korea
}

\begin{abstract}
Background: CDKN2A is a tumor suppressor gene that encodes the cell cycle inhibitor protein p16. Homozygous deletion of the CDKN2A gene has been associated with shortened survival in isocitrate dehydrogenase (IDH)-mutant gliomas. This study aimed to analyze the prognostic value of $\mathrm{p} 16$ and to evaluate whether 16 immunohistochemical staining could be used as a prognostic marker to replace CDKN2A genotyping in diffuse gliomas. Methods: p16 immunohistochemistry was performed on tissue microarrays of 326 diffuse gliomas with diagnoses that reflected IDH-mutations and $1 \mathrm{p} / 19 \mathrm{q}$ codeletion status. The results were divided into three groups (negative, focal expression, overexpression) according to the presence and degree of p16 expression. Survival analysis was performed to assess the prognostic value of $\mathrm{p} 16$ expression. Results: A loss of $\mathrm{p} 16$ expression predicted a significantly worse outcome in all glioma patients $(n=326, p<.001)$, in the IDH-mutant glioma patients $(n=103, p=.010)$, and in the IDH-mutant astrocytoma patients $(n=73, p=.032)$. However, loss of $\mathrm{p} 16$ expression did not predict the outcome in the IDH-wildtype glioma patients $(n=223, p=.121)$ or in the oligodendroglial tumor patients with the IDH-mutation and $1 p / 19 q$ codeletion $(n=30, p=.457)$. Multivariate analysis showed the association was still significant in the IDH-mutant glioma patients ( $p=.008$; hazard ratio [HR], 2.637; 95\% confidence interval [Cl], 1.295 to 5.372 ) and in the IDH-mutant astrocytoma patients ( $p=.001 ; \mathrm{HR}, 3.586 ; 95 \% \mathrm{Cl}, 1.649$ to 7.801 ). Interestingly, patients who presented with tumors with $\mathrm{p} 16$ overexpression also had shorter survival times than did patients with tumors with $\mathrm{p} 16$ focal expression in the whole glioma $(p<$ .001 ) and in IDH-mutant glioma groups. ( $p=.046)$. Conclusions: This study suggests that detection of p16 expression by immunohistochemistry can be used as a useful surrogate test to predict prognosis, especially in IDH-mutant astrocytoma patients.
\end{abstract}

Key Words: Glioma; p16; Immunohistochemistry; Prognosis; CDKN2A

Received: October 8, 2020 Revised: October 20, 2020 Accepted: October 22, 2020

Corresponding Author: Sung-Hye Park, MD, PhD, Department of Pathology, Seoul National University College of Medicine, 103 Daehak-ro, Jongno-gu, Seoul 03080, Korea Tel: +82-2-740-3090, Fax: +82-2-743-5530, E-mail: shparknp@snu.ac.kr

CDKN2A is a tumor suppressor gene located on chromosome 9p21 that encodes the cell cycle inhibitor protein p16 [1]. Genetic alterations of this gene are frequently observed in various types of human cancers [1-3]. With regard to brain tumors and prognosis, homozygous deletion of the CDKN2A gene has been reported to be associated with shortened survival in isocitrate dehydrogenase $(I D H)$-mutant glioma patients [4-8]. In the recent expert meeting of cIMPACT-NOW (the Consortium to Inform Molecular and Practical Approaches to CNS Tumor Taxonomy-Not Official WHO), update 5, genetic testing for the detection of CDKN2A homozygous deletion has been recommended for grade IDH-mutant astrocytic tumors. Its importance is increasingly high in adult and pediatric glioma patients [4,6,9-11].
Molecular testing to identify the $C D K N 2 A$ deletion requires advanced, high-cost equipment. However, the results of molecular testing are difficult to interpret. Therefore, it is challenging to use molecular testing in routine diagnostic practice. Since the CDKN2A gene product is the p16 protein, immunohistochemical detection of p16 protein expression can be used instead of molecular testing to identify the CDKN2A gene deletion. Before 2010 , in the pre- $I D H$-era, a few studies investigated the predictive value of p16 immunoreactivity in glioma samples [12-17]. The studies mainly targeted heterogenous groups of gliomas that did not reflect the current molecular genetics integrated classification. The sample size was small, and the results were not consistently conclusive. In addition, no large-scale comprehensive studies have been conducted on the prognostic significance of 
p16 immunochemistry in gliomas, particularly in this $I D H$-era of brain tumor diagnoses.

Meanwhile, although p16 is a tumor suppressor, aberrant overexpression of p16 protein has been observed in several tumors, including uterine cervical cancer, breast cancer, colorectal adenocarcinoma, and malignant melanoma [18-20]. In these tumors, p16 overexpression occurs by various mechanisms and has diverse prognostic implications depending on the tumor type [21]. Therefore, in addition to examining the prognostic relevance of p16 protein loss, it is also worth evaluating the prognostic implications of 16 overexpression in glioma patients.

This study aimed to determine whether p16 immunohistochemical staining can be used as a prognostic marker to replace $C D K N 2 A$ genotyping in molecularly characterized diffuse gliomas. We first examined the correlation and concordance between p16 immunochemistry and CDKN2A fluorescent in situ hybridization (FISH) results. In addition, considering that p16 is a negative regulator of cell proliferation, we evaluated whether the loss of p16 expression was related to an increase in the cell proliferation marker Ki-67 labeling index. Finally, the survival analyses were performed to evaluate the prognostic implications of p16 protein expression patterns in the whole sample, and in the subgroups according to $I D H$-mutation and $1 \mathrm{p} / 19 \mathrm{q}$ codeletion status.

\section{MATERIALS AND METHODS}

\section{Subjects and data acquisition}

A total of 326 cases of diffuse glioma from Seoul National University Hospital (SNUH) between 2011 and 2015 with tissue microarray (TMA) blocks were included in this study. The clinical information and test results that were previously conducted to assist in diagnosis were obtained retrospectively from the electronic medical records of SNUH. The overall survival time was calculated from the date of surgery to the date of death. All diagnoses were reassessed to reflect genetic alterations, including $I D H$-mutation and $1 \mathrm{p} / 19 \mathrm{q}$ codeletion status according to the cIMPACT-NOW update 5 and 6, and the 2016 World Health Organization (WHO) classification of central nervous system tumors.

\section{Evaluation of p16 immunohistochemistry}

The immunohistochemical staining for p16 was conducted on the TMA sections using an antibody against p16 (mouse monoclonal, clone E6H4, Ventana, Tucson, AZ, USA) and the Ventana BenchMark XT automated immunohistochemical staining sys- tem following the manufacturer's protocol. The results of immunohistochemical staining were first scored as the percentage of positively stained cells. Tumor cells with only nuclear or concurrent nuclear and cytoplasmic immunoreactivity were considered positive. The scoring was performed blindly without any knowledge of the diagnostic or clinical information. If there were no positive cells, or if the percentage of positive cells was $<1 \%$, then the tumor was classified as p16 negative (loss of expression). Conversely, tumors with more than $1 \%$ immunopositivity were considered to be p16 positive (retained expression). The p16 immunopositive cases were further divided into focal expression (if the positive cells were $<50 \%$ ) or diffuse overexpression (if the positive cells were $>50 \%$ ) according to the degree of p16 expression. Notably, in the group with p16 overexpression, most of the tumor cells showed simultaneous intense nuclear and cytoplasmic staining (Fig. 1).

\section{Other immunohistochemical and molecular testing variables}

The previously used antibodies included IDH1 (1:100, mouse monoclonal, clone H09, Dianova, Hamburg, Germany), p53 (1:1,000, mouse monoclonal, clone DO-7, DAKO, Glostrup, Denmark), PTEN (1:400, mouse monoclonal, clone 6H2.1, DAKO) and Ki-67 (1:100, mouse monoclonal, clone MIB-1, DAKO). Quantification of the Ki-67 labeling index was determined by the Nuclear v9 algorithm using the Aperio ImageScope software (Aperio Technologies, Vista, CA, USA). p53 was considered to be overexpressed if $\geq 30 \%$ of the tumor nuclei showed robust immunopositivity [22]. For IDH1 and PTEN, the immunohistochemical results were evaluated as positive or negative. Positive IDH1 immunostaining indicated the presence of a mutation, while negative immunostaining of PTEN meant protein loss.

Molecular tests that were previously performed for diagnosis included 1p/19q FISH to detect chromosome $1 \mathrm{p}$ and $19 \mathrm{q}$ codeletion; IDH1/IDH2 direct sequencing (performed only when the IDH1 immunohistochemistry result was negative); CDKN2A (9p21) FISH to catch gene deletion; epidermal growth factor receptor (EGFR) (7p12) FISH to detect gene amplification; PTEN (10q23) FISH to detect gene deletion; and $\mathrm{O}^{6}$-methylguanineDNA methyltransferase (MGMT) methylation-specific polymerase chain reaction analysis. The probes used to perform FISH were as follows: Vysis LSI 1p36 SpectrumOrange/1q25 SpectrumGreen Probes and Vysis LSI 19q13 SpectrumOrange/ 19p13 SpectrumGreen Probes (Abbott Molecular, Vysis, Des Plaines, IL, USA); Vysis LSI CDKN2A SpectrumOrange/CEP 

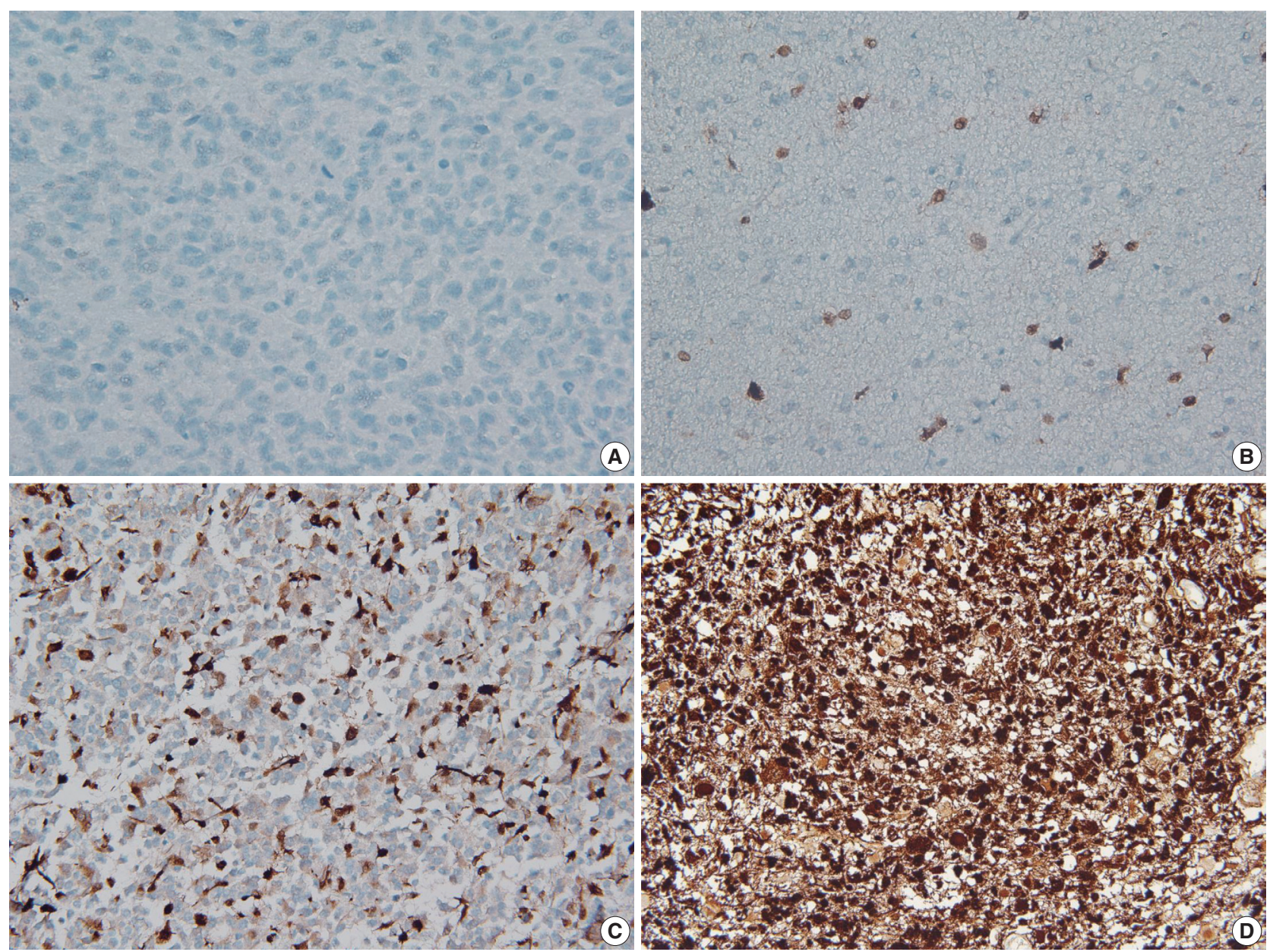

Fig. 1. Representative images of p16 immunohistochemical staining. If immunopositive cells were absent or made up $<1 \%$ of cells, the tumor was classified as having a loss of expression (A). Conversely, tumors with $>1 \%$ of immunopositivity were considered to have retained expression, which was further subdivided into focal expression $(B, C)$ or overexpression (D) according to the degree of p16 expression on a $50 \%$ basis. $\mathrm{B}$ and $\mathrm{C}$ show the range of focal expression.

9 SpectrumGreen Probes (Abbott Molecular); Vysis LSI EGFR SpectrumOrange/CEP 7 SpectrumGreen Probes (Abbott Molecular); Vysis LSI PTEN/CEP 10 Dual Color Probe (Abbott Molecular). Each molecular test was performed and interpreted as previously described $[23,24]$. Homozygous deletion of the $C D$ $K N 2 A$ gene was determined when $>15 \%$ of tumor cells lost two test signals in the presence of at least one reference signal in 100 non-overlapping counted nuclei [25]. PTEN is a representative quasi-sufficient and obligate haploinsufficient tumor suppressor gene [26]. A PTEN hemizygous deletion was considered if $>50 \%$ of tumor cells showed more than one test signal loss or if $>10 \%$ of tumor cells showed two test signal losses in 100 non-overlapping nuclei with two reference signals [27]. A PTEN homozygous deletion was defined by the loss of both PTEN signals in more than $30 \%$ of tumor cells when counting more than 100 non-overlapping nuclei with at least one reference signal [27]. With regard to the PTEN evaluation, most cases were evaluated by only one of the PTEN FISH or PTEN immunohistochemistry. The final results related to PTEN loss were used in combination with the two tests.

\section{Statistical analyses}

The chi-square test was used to examine the association between categorical variables. Cohen's kappa coefficient $(\kappa)$ was calculated to determine whether there was a concordance between p16 immunochemistry and CDKN2A FISH. The Mann-Whitney U-test was used to test the relationship between p16 immunoreactivity and the Ki-67 labeling index. Kaplan-Meier survival curves were generated to estimate the overall survival distributions. The log-rank test was used for univariate survival comparisons. The Cox proportional hazards regression model was applied for the multivariate analyses using forward stepwise variable se- 
lection. Variables with prognostic significance in univariate analyses were selected as independent variables in the multivariate analysis model. Statistical significance was defined as a p-value of $<.05$. All statistical analyses were performed using IBM SPSS Statistics software ver. 26.0 (IBM Corp., Armonk, NY, USA).

\section{RESULTS}

\section{Characteristics of the study population}

The median age of the study population was 54 years (range, 16 to 82 years), and 185 patients (56.7\%) were men. Sixty-five cases $(19.9 \%)$ were of recurrent tumors. Most patients were treated with surgery, followed by adjuvant radiotherapy and/or chemotherapy. Detailed clinical information is shown in Table 1. For the survival data, the median follow-up time was 23 months (range, 0 to 103 months). During this follow-up period, 220 patients (67.5\%) died, while 106 patients (32.5\%) were still alive at the last contact. The diagnoses according to cIMPACT-NOW update 5 and 6 and 2016 WHO classification were as follows: astrocytoma, $I D H$-mutant, $\mathrm{WHO}$ grade $2(\mathrm{n}=8,2.5 \%)$; anaplastic astrocytoma, $I D H$-mutant, WHO grade $3(\mathrm{n}=27,8.3 \%)$; astrocytoma, IDH-mutant, WHO grade $4(\mathrm{n}=38,11.7 \%)$; oligodendroglioma, $I D H$-mutant and 1p/19q-codeleted (ODG), WHO grade $2(\mathrm{n}=12,3.7 \%)$; ODG, WHO grade $3(\mathrm{n}=18$, 5.5\%); diffuse astrocytoma, $I D H$-wildtype ( $\mathrm{n}=3,0.9 \%)$; anaplastic astrocytoma, $I D H$-wildtype $(\mathrm{n}=25,7.7 \%)$; and glioblastoma, $I D H$-wildtype $(\mathrm{n}=195,59.8 \%)$. Overall, there were 103 $\mathrm{IDH}$-mutant tumors and $223 \mathrm{IDH}$-wildtype tumors. p16 loss was more prevalent in the $I D H$-wildtype tumors than it was in the $I D H$-mutant tumors (IDH-wildtype, 120 out of 223 cases [53.8\%] vs. IDH-mutant, 23 out of 103 cases [22.3\%]; $<<.001$ ). Among the $I D H$-mutant tumors, the frequency of p16 loss was similar between the $I D H$-mutant astrocytomas $(23.3 \%, 17$ out of 73 cases) and ODG (20\%, 6 out of 30 cases) $(\mathrm{p}=.716)$. The age of the patients was significantly higher in the p16 loss group than it was in the p16 expression group (p16 loss group, median 56 [range, 16 to 82] vs. p16 expression group, median 50 [range, 17 to 77]; $\mathrm{p}<0.001$ ). There was no significant association between p16 expression status and other clinical variables such as sex, the extent of surgery (biopsy or resection), recurrence, or adjuvant treatment. The immunohistochemical and FISH results of the study population are presented in Table 2.

\section{Comparison of p16 immunochemistry and CDKN2A FISH results}

The association between the CDKN2A homozygous deletion
Table 1. Clinicopathologic information

\begin{tabular}{|c|c|}
\hline Characteristic & No. $(\%)(n=326)$ \\
\hline Age (yr) & $54(16-82)$ \\
\hline \multicolumn{2}{|l|}{ Sex } \\
\hline Male & $185(56.7)$ \\
\hline Female & $141(43.3)$ \\
\hline \multicolumn{2}{|l|}{ IDH status } \\
\hline $\mathrm{IDH}$-mutant & $103(31.6)$ \\
\hline IDH-wildtype & $223(68.4)$ \\
\hline Astrocytoma, IDH-mutant ${ }^{\mathrm{a}}$ & $73(22.4)$ \\
\hline Astrocytoma, IDH-mutant, WHO grade 2 & $8(2.5)$ \\
\hline Astrocytoma, IDH-mutant, WHO grade 3 & $27(8.3)$ \\
\hline Astrocytoma, IDH-mutant, WHO grade 4 & $38(11.7)$ \\
\hline ODG ${ }^{a}$ & $30(9.2)$ \\
\hline ODG, WHO grade 2 & $12(3.7)$ \\
\hline ODG, WHO grade 3 & $18(5.5)$ \\
\hline Glioma, IDH-wildtype ${ }^{a}$ & $223(68.4)$ \\
\hline Diffuse astrocytoma, IDH-wildtype & $3(0.9)$ \\
\hline Anaplastic astrocytoma, IDH-wildtype & $25(7.7)$ \\
\hline Glioblastoma, IDH-wildtype & $195(59.8)$ \\
\hline \multicolumn{2}{|l|}{ Survival outcome } \\
\hline Death & $220(67.5)$ \\
\hline Censored & $106(32.5)$ \\
\hline Survival time (mo), median (range) & $23(0-103)$ \\
\hline \multicolumn{2}{|l|}{ Extent of surgery } \\
\hline Biopsy & $22(6.7)$ \\
\hline Resection & $304(93.3)$ \\
\hline \multicolumn{2}{|l|}{ Tumor recurrence } \\
\hline Primary tumor & $261(80.1)$ \\
\hline Recurred tumor & $65(19.9)$ \\
\hline \multicolumn{2}{|l|}{ Adjuvant treatment $(\mathrm{n}=323)$} \\
\hline Yes & 300 (92.9) \\
\hline No & $23(7.1)$ \\
\hline
\end{tabular}

IDG, isocitrate dehydrogenase; WHO, World Health Organization; ODG, oligodendroglioma, IDH-mutant and 1p/19q-codeleted.

aDiagnoses were made by cIMPACT-Now update 5 and 6, and 2016 World Health Organization classification.

and the loss of p16 expression was significant $(\mathrm{p}<.001)$ (Table 3). Sixty-eight percent (88/129) of tumors with CDKN2A homozygous deletions by FISH demonstrated a loss of p16 expression by immunohistochemistry, while $72 \%$ (142/197) of tumors without the CDKN2A homozygous deletion showed p16 immunopositivity. Therefore, the diagnostic accuracy of p16 immunochemistry to detect a $C D K N 2 A$ homozygous deletion confirmed by FISH was $70.6 \%$ (250/326). There was fair agreement between the CDKN2A FISH and p16 immunohistochemistry results (Cohen's kappa $=0.396, \mathrm{p}<.001)$.

\section{Correlation between p16 immunohistochemistry and Ki-67} labeling index

There was a significant inverse correlation between p16 expression and the Ki-67 labeling index $(\mathrm{p}<.001)$. That is, tumors with 
p16 loss had a significantly higher Ki-67 labeling index (mean, $27.60 \%$; median, $22.50 \%$; range, 1.11 to 90.16 ) than did those with p16 expression (mean, 19.98\%; median, $12.90 \%$; range, 0.40 to 74.46$)$. In addition, when the p16 retained expression (no loss) group was divided into two additional groups of overexpression and focal expression, the p16 overexpression group (mean, 27.73\%; median, 24.70\%; range, 2.34 to 72.70 ) showed a significantly higher $\mathrm{Ki}-67$ labeling index than did the p16 focal expression group (mean, 16.30\%, median, 8.55\%; range,

Table 2. Immunohistochemical and FISH results of the included cases

\begin{tabular}{lc}
\hline Parameter & No. (\%) \\
\hline p16 IHC & $143(43.9)$ \\
Loss & $183(56.1)$ \\
No loss (retained) & $124(38.0)$ \\
$\quad$ Focal expression & $59(18.1)$ \\
$\quad$ Overexpression & \\
CDKN2A FISH & $129(39.6)$ \\
Homozygous deletion & $197(60.4)$ \\
No deletion & \\
EGFR amplification & $63(19.3)$ \\
Positive & $263(80.7)$ \\
Negative & \\
PTEN alteration ( $\mathrm{n}=324)$ & $41(12.6)$ \\
Loss & $283(86.8)$ \\
No loss & \\
p53 overexpression & $143(43.9)$ \\
Positive & $183(56.1)$ \\
Negative & \\
MGMTp methylation (n=324) & $182(55.8)$ \\
Positive & $142(43.6)$ \\
Negative & $19.41(0.4-90.16)$ \\
Ki-67 labeling index, median (range, \%)
\end{tabular}

FISH, fluorescence in situ hybridization; IHC, immunohistochemistry; EGFR, epidermal growth factor receptor; MGMTp, O6-methylguanine-DNA methyltransferase promoter.

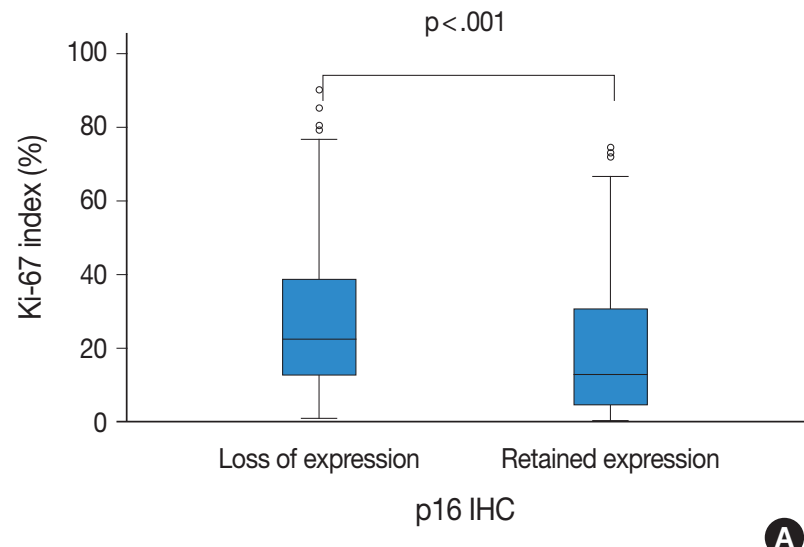

0.40 to $74.46 ; \mathrm{p}<.001)$. There was also a significant difference in the Ki-67 labeling index between the p16 focal expression group and the p16 loss of expression group (mean, 27.60\%; median, 22.50\%; range, 1.11 to 90.16; $\mathrm{p}<.001)$. However, there was no significant difference between $\mathrm{p} 16$ loss of expression and the 16 overexpression groups ( $\mathrm{p}=.940)$ (Fig. 2).

\section{Survival analysis}

In a total of 326 whole glioma samples, a loss of p16 expression was significantly associated with short overall survival in univariate analysis using Kaplan-Meier curves and the log-rank test ( $\mathrm{p}<.001)$ (Fig. 3A). When stratified by IDH status, tumors with p16 loss demonstrated a significantly worse outcome in $I D H$-mutant glioma patients ( $\mathrm{p}=.010)$ (Fig. 3B) than did those without p16 loss. However, no such association was found in the $I D H$-wildtype gliomas ( $\mathrm{p}=.121$ ) (Fig. 3C). Other parameters whose prognostic significance was confirmed by the log-rank test included recurrent tumors ( $\mathrm{p}<.001), E G F R$ amplification $(\mathrm{p}=$ $.004)$ and $\mathrm{p} 53$ overexpression $(\mathrm{p}=.002)$ in $I D H$-mutant glioma patients. In contrast, the extent of surgery $(\mathrm{p}=.043)$, adjuvant treatment $(\mathrm{p}=.005)$ and $M G M T$ promoter methylation $(\mathrm{p}=$ .024) were included in the IDH-wildtype gliomas. Sex and PTEN loss had no significant prognostic association with either group.

Table 3. Comparison between CDKN2A FISH and p16 immunohistochemistry

\begin{tabular}{lccccc}
\hline \multirow{2}{*}{ p16 IHC } & \multicolumn{2}{c}{ CDKN2A FISH } & Total & p-value ${ }^{\mathrm{a}}$ \\
\cline { 2 - 3 } & Deletion & No deletion & & \\
\hline Loss & $88(68.2)$ & $55(27.9)$ & 143 & $<.001$ \\
No loss & $41(31.8)$ & $142(72.1)$ & 183 & \\
Total & $129(100)$ & $197(100)$ & 326 & \\
\hline
\end{tabular}

Values are presented as number (\%).

FISH, fluorescence in situ hybridization; IHC, immunohistochemistry. ${ }^{a} \mathrm{p}$-value was determined using chi-square test.

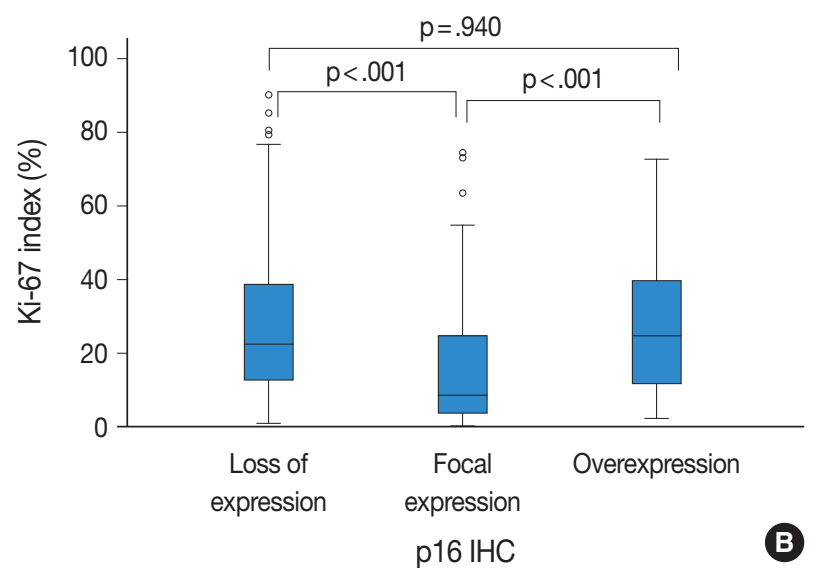

Fig. 2. Ki-67 labeling index according to p16 expression status (A) and the degree of p16 expression (B). IHC, immunohistochemistry. 

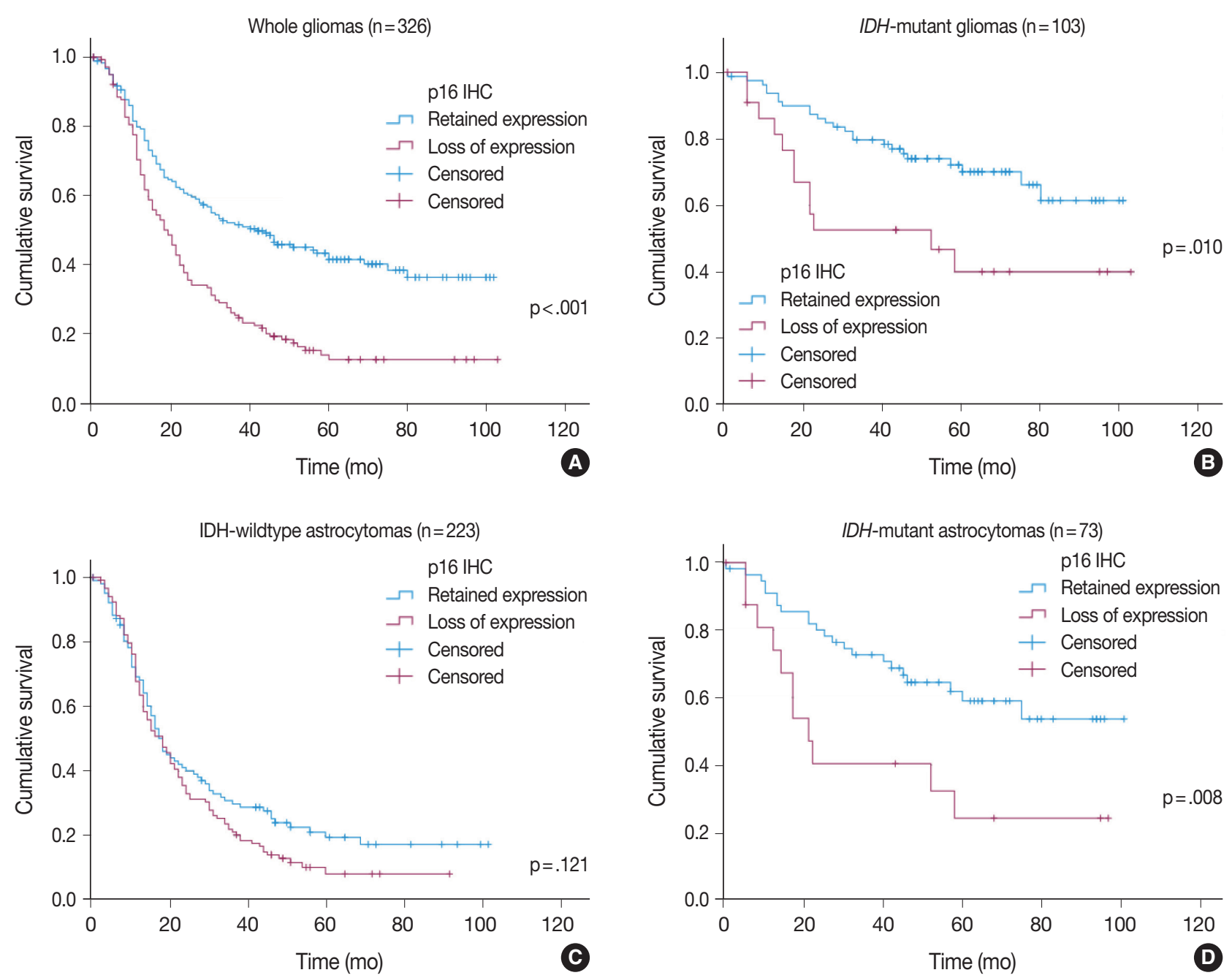

Fig. 3. Kaplan-Meier curves for overall survival according to p16 expression status: (A) whole gliomas, (B) IDH-mutant gliomas including oligodendrogliomas, (C) IDH-wildtype astrocytomas, and (D) IDH-mutant astrocytomas. IDH, isocitrate dehydrogenase; IHC, immunohistochemistry.

Table 4. Multivariate Cox regression analysis

\begin{tabular}{|c|c|c|c|c|c|c|}
\hline \multirow{2}{*}{ Variable } & \multicolumn{3}{|c|}{ IDH-mutant gliomas $(n=103)$} & \multicolumn{3}{|c|}{ IDH-mutant astrocytomas $(n=73)$} \\
\hline & $\mathrm{HR}$ & $95 \% \mathrm{Cl}$ & p-value & $\mathrm{HR}$ & $95 \% \mathrm{Cl}$ & $\mathrm{p}$-value \\
\hline p16 loss $(\mathrm{HC})$ & 2.637 & $1.295-5.372$ & .008 & 3.586 & $1.649-7.801$ & .001 \\
\hline Recurred tumor & 3.404 & $1.721-6.732$ & $<.001$ & 4.398 & $2.111-9.165$ & $<.001$ \\
\hline p53 overexpression & 2.725 & $1.371-5.417$ & .004 & NA & NA & .479 \\
\hline
\end{tabular}

$\mathrm{IDH}$, isocitrate dehydrogenase; IHC, immunohistochemistry; HR, hazards ratio; $\mathrm{Cl}$, confidence interval; NA, not applicable.

Multivariate Cox proportional hazards regression analysis was performed on the $I D H$-mutant gliomas using variables selected in univariate analyses as covariates. Although CDKN2A FISH and $\mathrm{Ki}-67$ were significantly prognostic for $\mathrm{IDH}$-mutant gliomas in univariate analysis $(\mathrm{p}=.001$, the log-rank test for $C D K N 2 A$ FISH; $\mathrm{p}<.001$, and univariate Cox regression analysis for Ki-67), they were not included in the final multivariate model because they were collinear with p16 immunohistochemistry. After ad- justing for recurrence, EGFR amplification and p53 overexpression, p16 loss was still a significant prognostic factor for worse outcome ( $\mathrm{p}=.008$; hazards ratio [HR], 2.637; 95\% confidence interval [CI], 1.295 to 5.372) (Table 4). Next, when the IDHmutant group was subdivided according to $1 \mathrm{p} / 19 \mathrm{q}$ codeletion status, loss of p16 expression was associated with significantly shorter overall survival in astrocytoma, IDH-mutant patients by the log-rank test $(\mathrm{p}=.008)$ (Fig. 3D), but not in ODG patients 
$(\mathrm{p}=.457)$. This association with the IDH-mutant astrocytomas was also significant in a multivariate analysis adjusted for recurrence, EGFR amplification, and p53 overexpression ( $\mathrm{p}=.001$; HR, 3.586; $95 \%$ CI, 1.649 to 7.801) (Table 4).

We also divided the p16 retained expression (no loss) tumors into two additional categories (focal expression vs. overexpression) according to the degree of p16 expression to check whether p16 overexpression has a prognostic meaning. Interestingly, among the 326 whole glioma cases, the p16 overexpression group showed a significantly worse overall survival curve than did the p16 focal expression group in Kaplan-Meier analysis ( $<$.001) (Fig. 4A). When limited to the IDH-mutant tumors (including astrocytomas and ODG), the p16 overexpression group had worse overall survival than did the p16 focal expression group ( $\mathrm{p}=.046)$ (Fig. 4B). This association was not seen in the $I D H$-wildtype

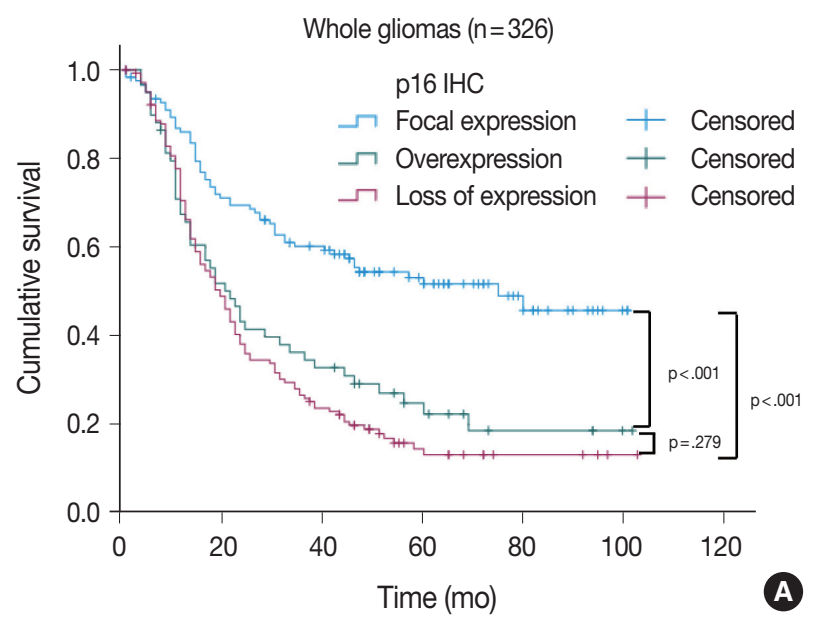

IDH-wildtype astrocytomas $(n=223)$

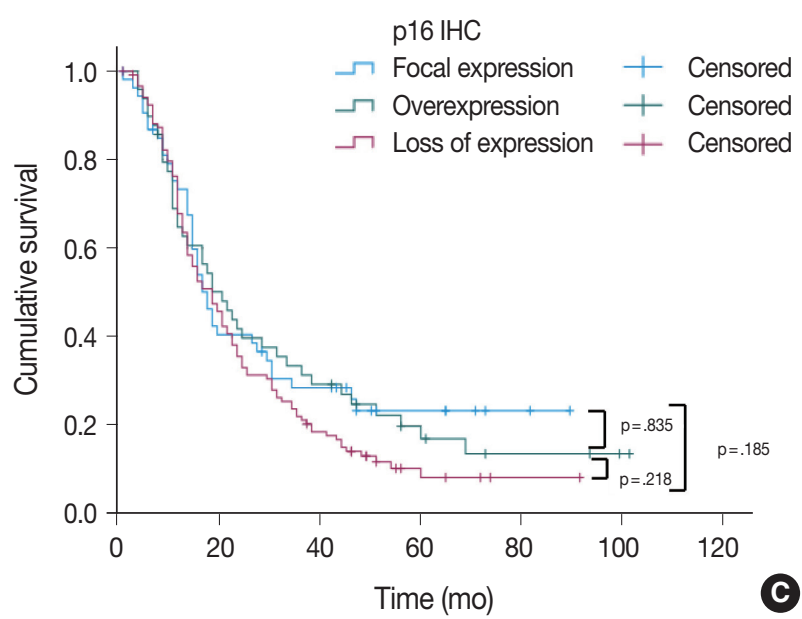

glioma patients (Fig. 4C). In multivariate analysis (adjusted for recurrence, p53 overexpression, and EGFR amplification), p16 overexpression was still associated with shorter overall survival than p16 focal expression, but this was not statistically significant ( $\mathrm{p}=.171 ; \mathrm{HR}, 2.048 ; 95 \% \mathrm{CI}, 0.734$ to 5.711$)$. In the subgroup of $I D H$-mutant astrocytoma patients, p16 overexpression also appeared to be related to shorter overall survival compared to p16 focal expression; however, the difference did not reach statistical significance in Kaplan-Meier analysis ( $\mathrm{p}=.251$ ) (Fig. 4D). Meanwhile, in the ODG, the analysis was not possible because none of the 30 cases showed p16 overexpression.

\section{DISCUSSION}

This study demonstrates the prognostic value of p16 immu-
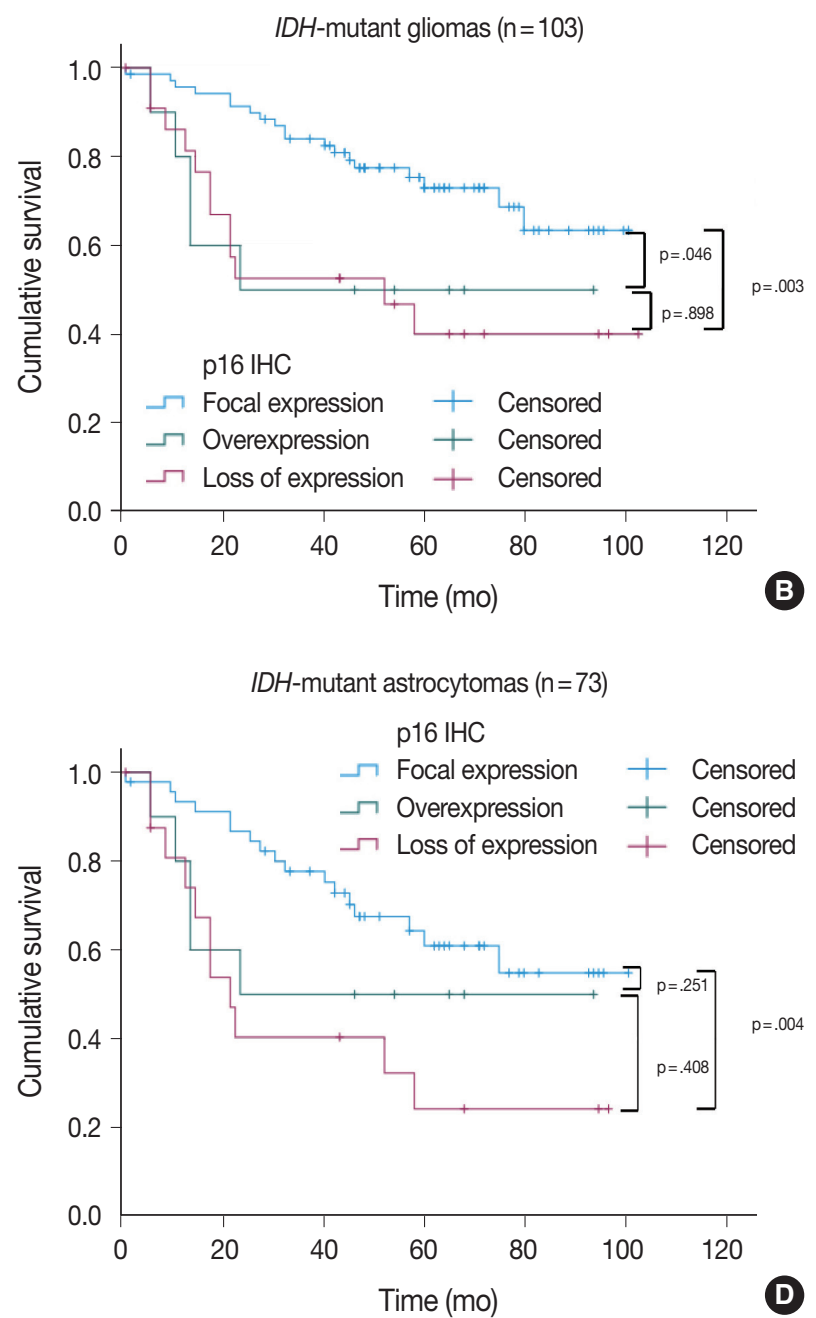

Fig. 4. Kaplan-Meier curves for overall survival according to the degree of p16 expression: (A) whole gliomas, (B) IDH-mutant gliomas including oligodendrogliomas, (C) IDH-wildtype astrocytomas, and (D) IDH-mutant astrocytomas. IHC, immunohistochemistry; IDH, isocitrate dehydrogenase. 
nohistochemical staining in a large sample of molecularly characterized diffuse gliomas. Negative immunohistochemical staining of the p16 protein predicted worse overall survival in all glioma patients and in the IDH-mutant subgroup, especially in $\mathrm{IDH}$ mutant astrocytomas, after adjusting for other prognostic factors such as tumor recurrence, $\mathrm{p} 53$ overexpression, and EGFR amplification. Our results were expected in light of existing knowledge that the p16 protein is encoded by the CDKN2A gene [1] and that $C D K N 2 A$ homozygous deletion is a significant prognostic factor in $I D H$-mutant glioma patients [9,28]. In addition, when the p16 retained expression tumors were divided into two additional categories of overexpression and focal expression, the tumors with p16 overexpression also demonstrated worse outcomes compared to tumors with p16 focal expression in the whole gliomas and $I D H$-mutant gliomas including $I D H$-mutant astrocytomas and ODG. Considering that immunohistochemistry is a relatively simple and convenient test to be used in routine practice, assessing p16 protein expression patterns with immunohistochemical methods would be a useful way to predict glioma prognosis in the field.

Previously, several studies have examined the association between p16 immunohistochemistry and molecular tests such as PCR or FISH to detect CDKN2A deletions in glioma samples $[5,13,29-31]$. Some of them reported that the association was good, while others did not agree. Reis et al [5]. reported that p16 expression by immunohistochemistry correlated poorly with CDKN2A deletion by FISH, and suggested that FISH be used to evaluate $C D K N 2 A$ status. This study differed from ours because the p16 immunohistochemistry data were used as continuous variables. In addition, this group counted the total number of signals regardless of whether the deletion pattern was hemizygous or homozygous when assessing the CDKN2A deletion by FISH. The problem with using the p16 expression data as a continuous variable is that it may not reflect the effect of p16 acting nonlinearly, as shown in our current study. In this study, Ki-67 was high in both the p16 loss and p16 overexpression subgroups, which was also associated with poor survival in each subgroup. The prior study appears to be the only study that has examined the relationship between p16 immunohistochemistry and survival in glioma patients molecularly diagnosed with both $I D H$ and $1 \mathrm{p} / 19 \mathrm{q}$ information to date. This group also found that there was a weak but significant association between p16 immunonegativity and poor overall survival in 83 astrocytoma patients.

In our study, although there was a correlation between $C D K$ N2A FISH and p16 immunohistochemistry, the degree of agreement between the two tests was fair, or at most moderate. There- fore, our results did not show a sufficient value to suggest that substitution between the two tests is reasonable. Fifty-five out of 197 (27.9\%) tumors without CDKN2A homozygous deletion (determined by FISH) demonstrated a loss of p16 expression. In contrast, 41 out of 129 (31.8\%) tumors with CDKN2A homozygous deletion (by FISH) demonstrated p16 immunopositivity in the present study. Such discrepancy may come from the fact that the expression of the p16 protein is controlled not only by cytogenetic alterations, but also by other mechanisms such as point mutations or epigenetic regulations such as EZH2 mediated transcriptional repression [31]. Furthermore, immuno-positive cases with CDKN2A deletion by FISH may be due to a problem on the immunohistochemistry side, such as a hidden mixture of normal tissue. Alternatively, this result may also be due to a problem on the FISH side, such as a false-positive FISH result caused by partial hybridization failure, truncation artifacts, or a suboptimal cutoff value [32]. Similar false-positive FISH results were previously reported in another study on mesotheliomas [33], which were attributed to suboptimal hybridization of the FISH probes. False-positive FISH results may also be attributable to the heterogeneity of p16 immunostaining and $C D$ $K N 2 A$ deletions in the same tumor, as previously demonstrated in gliomas [34]. Given that FISH is relatively expensive, difficult to perform correctly, and requires a skilled technician, one must reconsider whether FISH is reliable and the gold standard of diagnosis. It is also noteworthy that p16 immunochemistry may function better as a prognostic marker than as a diagnostic one, because it reflects cases in which protein expression of the $C D$ $K N 2 A$ gene is suppressed by mechanisms other than deletion, such as epigenetic silencing or point mutations. In $I D H$-mutant gliomas, DNA methylation occurs frequently due to the so-called glioma CpG island methylator phenotype (G-CIMP) [35]. Detecting the absence of expressed proteins by immunohistochemistry may reflect a more ultimate situation than detecting the deletion of a gene by molecular testing.

In addition to p16 loss, we also examined whether the overexpression of p16 protein has any prognostic implications. Contrary to a cell cycle inhibitor's original function, p16 overexpressing tumors were found to have a high proliferation index as measured by Ki-67, and to have poor prognosis, especially in $I D H$ mutated tumors. p16 is a component of the cell cycle regulation pathway that converges into the tumor suppressor protein $\mathrm{Rb}$. Disruption of $\mathrm{Rb}$ results in $\mathrm{p} 16$ overexpression in cancer tissue due to positive feedback [21]. Nakamura et al. [36] found that loss of expression of the RB1 gene was common in secondary glioblastoma. Therefore, the p16 overexpressing tumors observed 
in our study may be tumors that have excessively increased their p16 level to compensate for the loss of Rb. Therefore, p16 overexpression seems to be a desperate effort to stop uncontrolled proliferation due to failure of the $\mathrm{Rb}$ pathway. Our finding that a high Ki-67 labeling index was observed in the p16 overexpression group is considered to be in good agreement with this situation. Therefore, for p16 overexpressing tumors, we must determine whether there is another genetic abnormality in the $\mathrm{Rb}$ pathway components that include $R B 1$ deletion or $C D K 4 / 6$ amplification, and whether this is associated with prognosis.

In the previous literature, there were inconsistent findings on whether a $C D K N 2 A$ homozygous deletion was associated with poor survival in $I D H$-mutant and 1p/19q-codeleted ODGs [4,8, 28]. Similarly, our study did not show any prognostic significance of p16 protein loss in ODG. However, our results must be interpreted with caution given the small sample of ODG cases and a relatively short follow-up period for lower-grade gliomas. Therefore, further research is likely needed. It was also significant that p16 overexpression was not observed in all of our study's 30 ODGs. More samples are needed to confirm that the ODGs do not have p16 overexpression.

A limitation of this study is that it was retrospective in nature. Therefore, the data collection was inevitably limited, and other factors that may be related to prognosis were not all included or excluded. Most importantly, the FISH data should be reevaluated. We were not able to review these data because the preserved FISH pictures were limited. In addition, we cannot rule out the possibility that the immunohistochemical readings were overestimated or underestimated if the tumor had heterogeneous p16 expression, because the analysis was performed using TMA slides. Meanwhile, p16 overexpression was a statistically significant prognostic factor, which was confirmed in univariate analysis of $I D H$ mutant gliomas including astrocytomas and ODG. This significance was concealed after multivariate analysis or after being divided into subgroups, which may be due to insufficient sample sizes and short follow-up. Therefore, larger studies are necessary.

In summary, this study demonstrated that the pattern of p16 expression was significantly correlated with the prognosis in $\mathrm{IDH}$ mutant glioma patients. p16 immunohistochemistry was correlated with CDKN2A FISH. The loss of p16 expression was strongly associated with shortened overall survival. In addition, the overexpression of p16 was also related to a worse outcome. We suggest that detecting p16 protein expression by immunohistochemistry could be used as a useful surrogate test or an initial screening assay to predict patient prognosis while replacing $C D K N 2 A$ genetic testing. Nevertheless, further studies in other cohorts may be required to confirm these results.

\section{Ethics Statement}

All procedures performed in the current study were approved by the Seoul National University Hospital Institutional Review Board (IRB No. H-2008111-1150) in accordance with the 1964 Helsinki declaration and its amendments. Formal written informed was waived for cases collected before February 2013. Otherwise, informed consent was obtained from each patient.

\section{ORCID}

Jin Woo Park https://orcid.org/0000-0003-0801-8208 Jeongwan Kang https://orcid.org/0000-0002-2111-2912 Ka Young Lim https://orcid.org/0000-0001-8240-6175 Hyunhee Kim https://orcid.org/0000-0003-3353-1628 Seong-Ik Kim https://orcid.org/0000-0001-7713-3782 Jae Kyung Won https://orcid.org/0000-0003-1459-8093 Chul-Kee Park https://orcid.org/0000-0002-2350-9876 Sung-Hye Park https://orcid.org/0000-0002-8681-1597

\section{Author Contributions}

Conceptualization: SHP, JWP. Data curation: JWP, SHP. Formal analysis: JWP. Funding acquisition: SHP. Investigation: JWP, JK, KYL, HK, SIK, JKW, SHP. Methodology: JWP, SHP. Resources: CKP. Supervision: SHP. Writing—original draft: JWP. Writing_review \& editing: JWP, SHP, JKW. Approval of final manuscript: all authors.

\section{Conflicts of Interest}

The authors declare that they have no potential conflicts of interest.

\section{Funding Statement}

This study was supported by a grant of the Korea Health Technology R\&D Project through the Korea Health Industry Development Institute (KHIDI), funded by the Ministry of Health \& Welfare, Republic of Korea (grant number: $\mathrm{HI} 14 \mathrm{C} 1277)$.

\section{References}

1. Foulkes WD, Flanders TY, Pollock PM, Hayward NK. The CDKN2A (p16) gene and human cancer. Mol Med 1997; 3: 5-20.

2. Serrano M. The tumor suppressor protein p16INK4a. Exp Cell Res 1997; 237: 7-13.

3. Raschke S, Balz V, Efferth T, Schulz WA, Florl AR. Homozygous deletions of CDKN2A caused by alternative mechanisms in various human cancer cell lines. Genes Chromosomes Cancer 2005; 42: 5867.

4. Appay R, Dehais C, Maurage CA, et al. CDKN2A homozygous deletion is a strong adverse prognosis factor in diffuse malignant $\mathrm{IDH}$ mutant gliomas. Neuro Oncol 2019; 21: 1519-28.

5. Reis GF, Pekmezci M, Hansen HM, et al. CDKN2A loss is associated with shortened overall survival in lower-grade (World Health Organization Grades II-III) astrocytomas. J Neuropathol Exp Neurol 2015; 74: 442-52.

6. Shirahata M, Ono T, Stichel D, et al. Novel, improved grading system(s) for $I D H$-mutant astrocytic gliomas. Acta Neuropathol 2018; 136: 153-66.

7. Yang RR, Shi ZF, Zhang ZY, et al. IDH mutant lower grade (WHO Grades II/III) astrocytomas can be stratified for risk by CDKN2A, CDK4 and PDGFRA copy number alterations. Brain Pathol 2020; 30: 541-53. 
8. Aoki K, Nakamura H, Suzuki H, et al. Prognostic relevance of genetic alterations in diffuse lower-grade gliomas. Neuro Oncol 2018; 20: 66-77.

9. Brat DJ, Aldape K, Colman H, et al. cIMPACT-NOW update 5: recommended grading criteria and terminologies for $\mathrm{IDH}$-mutant astrocytomas. Acta Neuropathol 2020; 139: 603-8.

10. Louis DN, Wesseling P, Aldape K, et al. cIMPACT-NOW update 6: new entity and diagnostic principle recommendations of the cIMPACT-Utrecht meeting on future CNS tumor classification and grading. Brain Pathol 2020; 30: 844-56.

11. Frazao L, do Carmo Martins M, Nunes VM, et al. BRAF V600E mutation and 9p21: CDKN2A/B and MTAP co-deletions: markers in the clinical stratification of pediatric gliomas. BMC Cancer 2018; 18: 1259 .

12. Miettinen H, Kononen J, Sallinen P, et al. CDKN2/p16 predicts survival in oligodendrogliomas: comparison with astrocytomas. J Neurooncol 1999; 41: 205-11.

13. Bortolotto S, Chiado-Piat L, Cavalla P, et al. CDKN2A/p16 inactivation in the prognosis of oligodendrogliomas. Int J Cancer 2000; 88: 554-7.

14. Kirla R, Salminen E, Huhtala S, et al. Prognostic value of the expression of tumor suppressor genes p53, p21, p16 and prb, and Ki67 labelling in high grade astrocytomas treated with radiotherapy. J Neurooncol 2000; 46: 71-80.

15. Jeon YK, Park K, Park CK, Paek SH, Jung HW, Park SH. Chromosome $1 \mathrm{p}$ and $19 \mathrm{q}$ status and p53 and p16 expression patterns as prognostic indicators of oligodendroglial tumors: a clinicopathological study using fluorescence in situ hybridization. Neuropathology 2007; 27: 10-20.

16. Puduvalli VK, Kyritsis AP, Hess KR, et al. Patterns of expression of $\mathrm{Rb}$ and $\mathrm{p} 16$ in astrocytic gliomas, and correlation with survival. Int J Oncol 2000; 17: 963-9.

17. Arifin MT, Hama S, Kajiwara Y, et al. Cytoplasmic, but not nuclear, p16 expression may signal poor prognosis in high-grade astrocytomas. J Neurooncol 2006; 77: 273-7.

18. Milde-Langosch K, Bamberger AM, Rieck G, Kelp B, Loning T. Overexpression of the p16 cell cycle inhibitor in breast cancer is associated with a more malignant phenotype. Breast Cancer Res Treat 2001; 67: 61-70.

19. O'Neill CJ, McCluggage WG. p16 expression in the female genital tract and its value in diagnosis. Adv Anat Pathol 2006; 13: 8-15.

20. Lam AK, Ong K, Giv MJ, Ho YH. p16 expression in colorectal adenocarcinoma: marker of aggressiveness and morphological types. Pathology 2008; 40: 580-5.

21. Romagosa C, Simonetti S, Lopez-Vicente L, et al. p16(Ink4a) overexpression in cancer: a tumor suppressor gene associated with se- nescence and high-grade tumors. Oncogene 2011; 30: 2087-97.

22. Chaurasia A, Park SH, Seo JW, Park CK. Immunohistochemical analysis of ATRX, IDH1 and p53 in glioblastoma and their correlations with patient survival. J Korean Med Sci 2016; 31: 1208-14.

23. Koh J, Cho H, Kim H, et al. IDH2 mutation in gliomas including novel mutation. Neuropathology 2015; 35: 236-44.

24. Kim SI, Lee Y, Won JK, Park CK, Choi SH, Park SH. Reclassification of mixed oligoastrocytic tumors using a genetically integrated diagnostic approach. J Pathol Transl Med 2018; 52: 28-36.

25. Chung CT, Santos Gda C, Hwang DM, et al. FISH assay development for the detection of p16/CDKN2A deletion in malignant pleural mesothelioma. J Clin Pathol 2010; 63: 630-4.

26. Berger AH, Knudson AG, Pandolfi PP. A continuum model for tumour suppression. Nature 2011; 476: 163-9.

27. Lotan TL, Wei W, Ludkovski O, et al. Analytic validation of a clinical-grade PTEN immunohistochemistry assay in prostate cancer by comparison with PTEN FISH. Mod Pathol 2016; 29: 904-14.

28. Lu VM, O'Connor KP, Shah AH, et al. The prognostic significance of $C D K N 2 A$ homozygous deletion in IDH-mutant lower-grade glioma and glioblastoma: a systematic review of the contemporary literature. J Neurooncol 2020; 148: 221-9.

29. Burns KL, Ueki K, Jhung SL, Koh J, Louis DN. Molecular genetic correlates of $\mathrm{p} 16, \mathrm{cdk} 4$, and $\mathrm{pRb}$ immunohistochemistry in glioblastomas. J Neuropathol Exp Neurol 1998; 57: 122-30.

30. Purkait $\mathrm{S}$, Jha $\mathrm{P}$, Sharma MC, et al. CDKN2A deletion in pediatric versus adult glioblastomas and predictive value of p16 immunohistochemistry. Neuropathology 2013; 33: 405-12.

31. Purkait S, Sharma V, Jha P, et al. EZH2 expression in gliomas: correlation with CDKN2A gene deletion/ p16 loss and MIB-1 proliferation index. Neuropathology 2015; 35: 421-31.

32. Fuller CE, Perry A. Fluorescence in situ hybridization (FISH) in diagnostic and investigative neuropathology. Brain Pathol 2002; 12: 67-86.

33. Chiosea S, Krasinskas A, Cagle PT, Mitchell KA, Zander DS, Dacic S. Diagnostic importance of 9p21 homozygous deletion in malignant mesotheliomas. Mod Pathol 2008; 21: 742-7.

34. Piva R, Cavalla P, Bortolotto S, et al. CDKN2/p16 inactivation and p16 immunohistochemistry in astrocytic gliomas. Int J Oncol 1998; 12: $55-8$.

35. Noushmehr H, Weisenberger DJ, Diefes K, et al. Identification of a CpG island methylator phenotype that defines a distinct subgroup of glioma. Cancer Cell 2010; 17: 510-22.

36. Nakamura M, Yonekawa Y, Kleihues P, Ohgaki H. Promoter hypermethylation of the RB1 gene in glioblastomas. Lab Invest 2001; 81: 77-82. 